ITC 4/50

Information Technology and Control

Vol. 50 / No. 4 / 2021

pp. '706-'721

DOI 10.5755/j01.itc.50.4.29484
Brightness Compensation for GF-3 SAR Images Based on Cycle-Consistent Adversarial Networks

Received 2021/07/21

Accepted after revision 2021/08/14

HOW TO CITE: Lin, S., Sun, Z.,Peng, X., Ni, L., Wen, G., Chen, W., Lu, Z., Chen, Q. (2021). Brightness Compensation for GF-3 SAR Images Based on Cycle-Consistent Adversarial Networks. Information Technology and Control, 50(4), 706-721. https://doi.org/10.5755/j01.itc.50.4.29484

\title{
Brightness Compensation for GF-3 SAR Images Based on Cycle- Consistent Adversarial Networks
}

\section{Shaofeng Lin}

College of Information and Communication, National University of Defense Technology, Xi'an Shaanxi 710106,

China; e-mail: 448537556@@qq.com

\section{Zengguo Sun}

Key Laboratory of Modern Teaching Technology, Ministry of Education, Xi'an Shaanxi 710062, China;

School of Computer Science, Shaanxi Normal University, Xi'an Shaanxi 710119, China

\section{Xuejun Peng}

School of Computer Science, Shaanxi Normal University, Xi'an Shaanxi 710119, China;

e-mail: pengxuejun@snnu.edu.cn

\section{Lin Ni}

College of Information and Communication, National University of Defense Technology, Xi'an Shaanxi 710106,

China; e-mail: nilin1008@sina.com

\section{Genfeng Wen}

School of Computer Science, Shaanxi Normal University, Xi'an Shaanxi 710119, China;

e-mail:m13632864226@163.com

\section{Weirong Chen}

China Centre for Resources Satellite Data and Application, Beijing 100094, China; e-mail: weirong49@163.com

\section{Zheng Lu}

Institute of Remote Sensing Satellite, Beijing 100094, China; e-mail: lvzheng_cast@163.com

\section{Qianfu Chen}

Land Satellite Remote Sensing Application Center, MNR, Beijing 100048, China; e-mail: chenqianfu-114@163.com

Corresponding author: Zengguo Sun (sunzg@snnu.edu.cn) 
GF-3 is the first C-Band full-polarimetric synthetic aperture radar (SAR) satellite with a space resolution up to $1 \mathrm{~m}$ in China. The uneven brightness of SAR images is a problem when using GF-3 images, which makes it difficult to use and produce SAR images. In this paper, a brightness compensation method is proposed for GF-3 SAR images with unbalanced brightness in some areas based on a deep learning model named Cycle-Consistent Adversarial Networks (CycleGAN). The proposed method makes the image brightness relatively consistent, and it is compared with the MASK dodging algorithm, Wallis dodging algorithm and histogram equalization in terms of the profiles, brightness mean, standard deviation, and average gradient. Results of brightness compensation show that, the proposed method makes the inner brightness differences smaller, and the image quality is obviously improved, which provides even brightness image for subsequent applications, and has great practical significance.

KEYWORDS: GF-3 synthetic aperture radar images, brightness compensation, Cycle-Consistent Adversarial Networks, deep learning.

\section{Introduction}

Since synthetic aperture radar (SAR) is a kind of microwave slant-range imaging radar, the amplitude information (or grayscale information and brightness information) of SAR images reflects the backscattering intensity of ground targets to radar waves [22, 32]. Complex texture features of terrain objects and imaging geometry make the amplitudes of SAR images have a large dynamic range, therefore, the radiations of SAR data acquired by different time phases and imaging modes are quite different, and the main manifestation is that the radiation (or brightness) of SAR image is not balanced. The uneven brightness of SAR images is a problem when using GF-3 images, which makes it difficult to use and produce SAR images. GF-3 satellite is not only the first $\mathrm{C}$ band and multi-polarization SAR satellite in China, but also the only microwave imaging satellite of the NHREOS (national high resolution earth observation system) $[39,40]$. During the period of making National Orthophoto Map of Radar Remote Sensing with GF-3 data [16], it is found that the data collected in the Taklimakan Desert area have the phenomenon of uneven brightness in close range and dark in long range. The examples of GF-3 uneven brightness images are shown in Figure 1.

With the rapid development of computer industry, people have made great breakthrough in the field of image processing. Scholars have a lot of researches on dodging algorithms (i.e. brightness compensation) of remote sensing images. According to the different principles and models of the dodging algorithms, the main algorithms can be divided into three categories [8]: the additive noise model algorithm, the illumination-reflectance model algorithm and the statistical method of the dodging algorithms. The additive noise
Figure 1

Examples of uneven brightness of GF-3 images

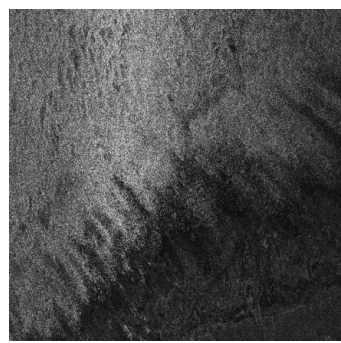

(a)

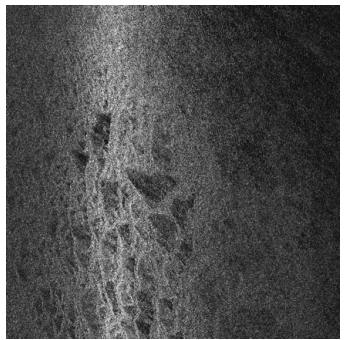

(b) model algorithm considers that the remote sensing image with uneven brightness distribution results from the remote sensing image with even brightness distribution and the noise image with uneven brightness distribution. The representative algorithm is MASK dodging algorithm [33]. It has a wide range of applications and can filter out most of the image details to obtain a better uniformity effect, but it is easy to produce halo around the image, resulting in color cast in the brightness compensation image. The dodging algorithm based on illumination-reflectance model considers that the remote sensing images are composed of the product of illuminance and scene reflectance. The classical algorithm is Retinex dodging algorithm [23]. It is mainly used to correct the uneven brightness in a single image, and the effect of dealing with the uneven illumination is better, but the contrast and clarity of the processed image are decreased to some extent. The dodging algorithm based on statistical method considers that the hue and brightness of remote sensing 
images are represented by their mean values, and the clarity and contrast of images are represented by their variances. The classic algorithm is Wallis filtering algorithm [9]. It has a very obvious effect on the brightness compensation of multiple images, but its effect on single image is not obvious, and there are "block effect", "hue shift" and other problems.

The above algorithms have their own scope of application. Since different dodging algorithms are different in scope and situation, it is necessary to select the corresponding algorithm according to the actual situation, so a single dodging algorithm is difficult to solve all problems at once. In recent years, deep learning technology has been widely and successfully applied in various fields [11, 13], such as target detection, super-resolution analysis and so on. In 2014, the Generative Adversarial Networks (GAN) proposed by Goodfellow [10] et al. gained the public's favor. It greatly promotes the development of unsupervised learning and saves a lot of data annotation costs. At the same time, it also provides a new solution for the lack of training set data and is known as the most promising unsupervised learning model [41]. As a result, GAN has developed rapidly, resulting in many excellent variant models based on GAN [3], such as info-GAN, DCGAN, etc. Among them, the Cycle-Consistent Adversarial Networks (CycleGAN) proposed by Jun-Yan Zhu [42] and others solves the problem that it is difficult to obtain pairs of training images in the field of image-to-image translation. We have made a deep research on CycleGAN and improved it. The image-to-image translation method is used to compensate the brightness of GF-3 SAR images, and its compensation effect is obviously better than the traditional dodging algorithms. The qualities of SAR images after brightness compensation are improved by using this method. Thus the method has a strong practical value in GF-3 data processing. As we all know, SAR transmits and receives radar waves through active imaging, and obtains images corresponding to ground targets through a series of signal processing methods. For SAR images, the phase information is very important, such as for SAR interferometry, SAR tomography and so on $[43,44]$. However, the images that need to be processed in this paper are all amplitude images without phase information. In other words, the amplitude images are directly compensated in order to obtain uniform brightness compensation results.

\section{Generative Adversarial Networks}

The generative adversarial network (GAN) is composed of generative model and discriminative model. For most of GAN [44], the discriminative model D is a traditional convolutional neural network, which extracts the features of the image through a series of convolution layers, normalization layers and activation functions to distinguish the true and false of the input image. Generative model $\mathrm{G}$ is a model composed of convolution neural network and deconvolution neural network, which is used to generate images. The input data of the generated model is random white Gaussian noise, and an image is output after encoding and decoding. The input data of the discriminative model is an image, and the discriminative model outputs a value between $[0,1]$ to represent the true and false probability of the input image. Generally, we label the images in training set as true images, and the images generated by the generated model as false images. In GAN, the generative model and the discriminative model learn from each other and finally produce good output, which can be expressed as follows [11,10].

$$
\begin{aligned}
& \min _{G} \max _{D} V(D, G)= \\
& E_{x \sim p_{\text {data }}(x)}[\log D(x)]+E_{z} \sim p_{z}(z)[\log (1-D(G(z)))]
\end{aligned} .
$$

It can be seen that GAN can obtain spurious datasets through input random noise. Therefore, GAN is often used for dataset enhancement to solve the problem of insufficient datasets [20]. At the same time, since GAN can generate analog images, it is also used in the field of image generation [12], such as semantic segmentation, super-resolution task and image-to-image translation. The example of SAR image generation using GAN model is shown in Figure 2. In this paper, we use the function of GAN image-to-image translation to realize brightness compensation (i.e. image smoothing).

\section{Figure 2}

Example of SAR image generation using GAN

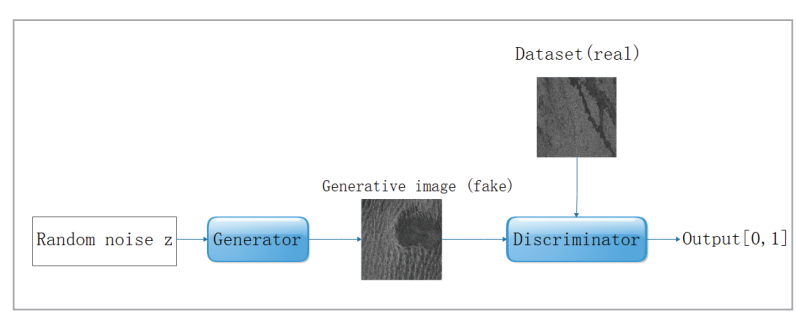




\section{CycleGAN}

CycleGAN is a general and strong GAN model. Unlike most of the GAN models, CycleGAN is a structurally symmetric GAN, and the input data of the generated model is images rather than random white Gaussian noise. Its main function is image-to-image translation. Image-to-image translation is a class of vision and graphics problem [38] and is to let the machine learn the mapping from class $X$ images to class $Y$ images, such as from gray images to color images and from contours to images. Before CycleGAN was put forward, many achievements have been achieved in the field of image-to-image translation [13, 4], such as pix2pix model, style transfer and so on. However, most image-to-image translation models require paired of training data. In other words, the class $X$ images in the training data must have corresponding class $Y$ images as labels. Obviously, in many image-to-image translation tasks, we cannot get paired training data, but it is relatively easy to get unpaired datasets. Figure 3 shows the example of paired and unpaired datasets.

\section{Figure 3}

Example of paired dataset and unpaired dataset: (a) paired dataset; (b) unpaired dataset

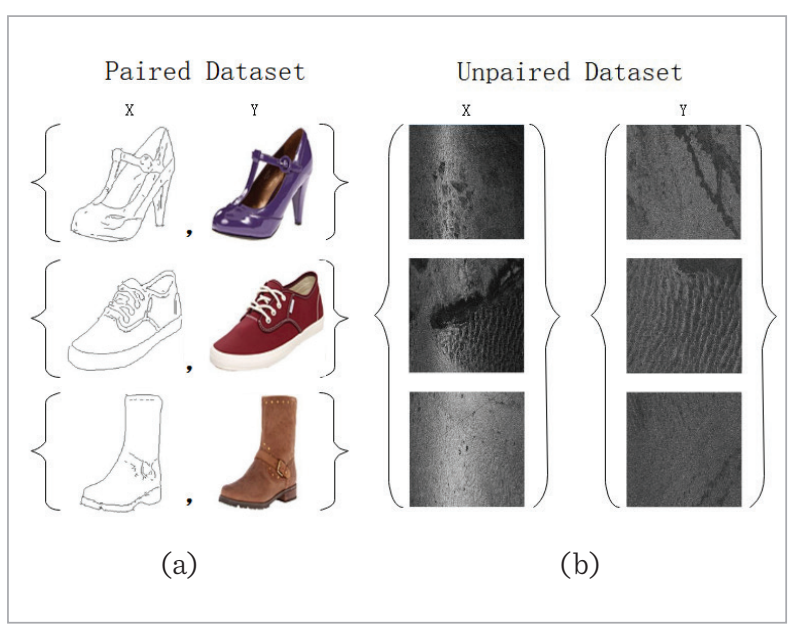

Aiming at the problem of brightness compensation, we cannot get an abnormal brightness image of a normal image, nor a normal image with abnormal brightness. Therefore, most of the image-to-image translation models are not suitable for brightness compensation. In the CycleGAN model, this prob- lem is well solved. In other words, the CycleGAN model can achieve image-to-image translation well without paired training data, and its practicability is stronger than the traditional image-to-image translation models.

There are two generators of $G_{X}$ and $G_{Y}$ and two discriminators of $D_{X}$ and $D_{Y}$ in the CycleGAN model. $G_{X}$ and $D_{Y}, G_{Y}$ and $D_{X}$ form a GAN, respectively. At the same time, the two GANs are symmetrical, and they form a ring network, which is the CycleGAN. Figure 4 is the schematic diagram of CycleGAN.

\section{Figure 4}

Schematic sketch of CycleGAN

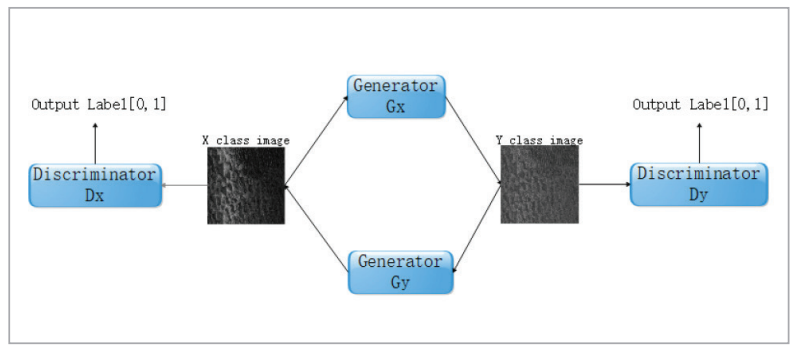

In the model, we have two kinds of images $X$ and $Y$. $X$ generates a fake class $Y$ image by generator $G_{X}$, that is $G_{X}(X)$. Meanwhile, the discriminator $D_{Y}$ determines whether the image $G_{X}(X)$ generated by generator is true or false. In the same way, $Y$ generates a fake class $X$ image through generator $G_{Y}$, that is $G_{Y}(Y)$. At the same time, discriminator $D_{X}$ determines whether the image $G_{Y}(Y)$ generated by generator is true or false. Therefore, the adversarial loss of $G_{x}$ and its discriminator $D_{Y}$ is as follows [10].

$$
\begin{aligned}
& L_{G A N}\left(G_{X}, D_{Y}, X, Y\right)= \\
& E_{y \sim p_{\text {data }}(y)}\left[\log D_{Y}(y)\right]+E_{x} \sim p_{\text {data }}(x)\left[\log \left(1-D_{Y}\left(G_{X}(x)\right)\right)\right]
\end{aligned}
$$

In the same way, the adversarial loss of $G_{y}$ and its discriminator $D_{X}$ is as follows.

$$
\begin{aligned}
& L_{G A N}\left(G_{Y}, D_{X}, Y, X\right)= \\
& E_{x \sim p_{\text {data }}(x)}\left[\log D_{X}(x)\right]+E_{y \sim p_{\text {data }}(y)}\left[\log \left(1-D_{X}\left(G_{Y}(y)\right)\right)\right] .
\end{aligned}
$$

Here, we explain the symbols of the above two formulas: $E_{x} \sim p_{\text {data }}(x)[f(x)]$ is the expectation of $f(x)$ about $p_{\text {data }}(x)$ when the random variable $x$ satisfies the $p_{\text {data }}$ probability distribution. 
Figure 5

Schematic sketch of cycle consistency

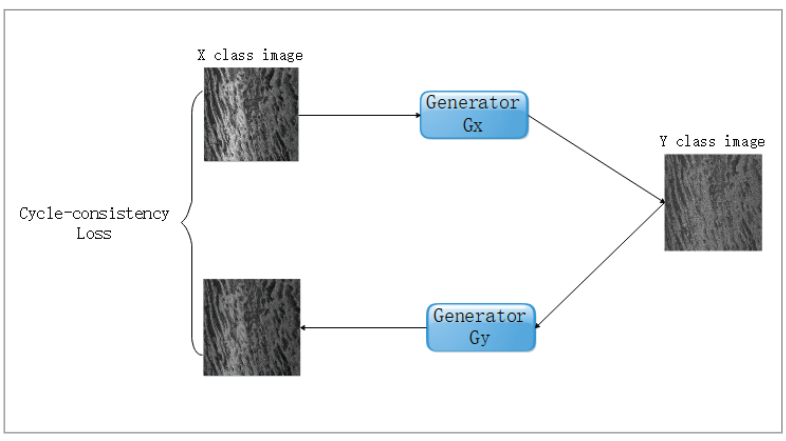

The formula (2) and (3) are exactly the same as the original GAN's adversarial losses. A major innovation of CycleGAN is the introduction of cycle consistency [42], and Figure 5 gives the schematic diagram of cycle consistency. The so-called cycle consistency means that in an ideal state, after the class $X$ image is translated into the class $Y$ image by the generator $G_{X}$, the class $Y$ image can be translated into the original class $X$ image by the generator $G_{Y}$, that is to say, the following formulas are met.

$$
\begin{aligned}
& G_{Y}\left(G_{X}(x)\right) \approx x \\
& G_{X}\left(G_{Y}(y)\right) \approx y
\end{aligned} .
$$

Satisfying the cycle consistency can avoid all class $X$ images mapping to the same class $Y$ image, thus the robustness of the CycleGAN model is guaranteed. Therefore, cycle consistency loss of the CycleGAN model is expressed as follows.

$$
\begin{aligned}
& L_{c y c}\left(G_{X}, G_{Y}\right)= \\
& E_{x} \sim P_{\text {data }}(x)\left[\left\|G_{Y}\left(G_{X}(x)\right)-x\right\|_{1}\right]+E_{y} \sim P_{\text {data }}(y)\left[\left\|G_{X}\left(G_{Y}(y)\right)-y\right\|_{1}\right]
\end{aligned}
$$

In a word, the objects of complete CycleGAN model are as follows, in which $\lambda$ is to control the relative importance of two objects.

$$
\begin{aligned}
& L\left(G_{X}, G_{Y}, D_{X}, D_{Y}\right)= \\
& L_{G A N}\left(G_{X}, D_{Y}, X, Y\right)+L_{G A N}\left(G_{Y}, D_{X}, Y, X\right)+\lambda L_{c y c}\left(G_{X}, G_{Y}\right)
\end{aligned} .
$$

According to the above model, the learning problem we need to solve is:

$$
G_{X}, G_{Y}=\arg \min _{G X, G Y} \max _{D X, D Y} L\left(G_{X}, G_{Y}, D_{X}, D_{Y}\right)
$$

\section{Brightness Compensation for SAR Images Based on CycleGAN}

\subsection{Architecture and Improvement of the Model}

Due to the great difference between SAR images and optical images, such as the particularity of imaging principle and accuracy requirements [5, 34], we have made some improvements on CycleGAN, so that the model has good image-to-image translation effect on SAR images.

The generator we built consists of three parts. They are introduced as follows.

1 Encoder: There are three convolution layers, the input channel is 1 . The output channel of the first convolution layer is 13 , the stride is 1 , the padding is SAME and the convolution kernel size is $7 * 7$. The output channel of the second convolution layer is 26, the stride is 2, the padding is SAME and the convolution kernel size is $3 * 3$. The output channel of the third convolution layer is 52 , the stride is 2 , the padding is SAME and the convolution kernel size is $3 * 3$. The activation functions are all ReLu.

2 Transformer: It is composed of several Resnet blocks and is the key step of image-to-image translation. The input channel and output channel are all 52, and the activation functions are all ReLu.

3 Decoder: There are two deconvolution layers and one convolution layer. The input channel is 52 . The output channel of the first deconvolution layer is 26 , the stride is 2 , the padding is SAME, and the convolution kernel size is $3 * 3$. The output channel of the second deconvolution layer is 13 , the stride is 2 , and the padding is SAME. Output channel of convolution layer is 1 , the stride is 1 , padding is SAME, and the convolution kernel size is $7 * 7$. The two deconvolution layers' activation functions are all ReLu, and convolution layer's activation function is Tanh. Decoder is used to restore the image to the input image size $(1024 * 1024)$ and output the image.

At the same time, we normalize each layer.

Figure 6 is a schematic diagram of the generator.

The discriminator we build is as follows. 
Figure 6

Schematic sketch of generator

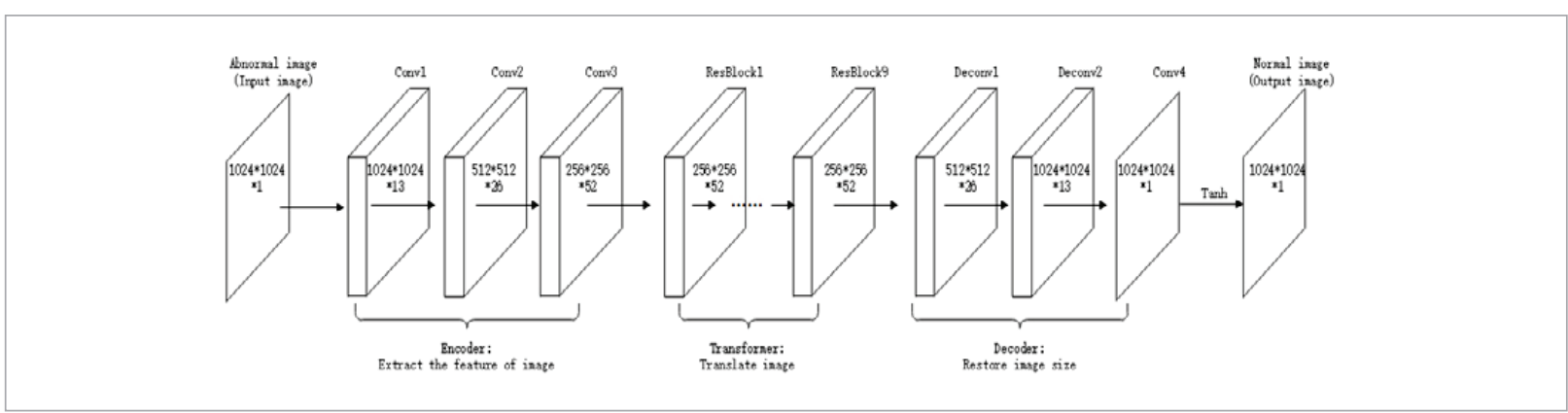

Using the patch strategy of the discriminator in Patch-GAN [27], the discriminator finally outputs a prediction map of $128 * 128 * 1$. The neural network model of the discriminator has five convolution layers and the input channel is 1 . The first convolution layer output channel is 13 , the stride is 2 , convolution kernel size is $4 * 4$ and padding is SAME. The second convolution layer output channel is 26 , stride is 2 , convolution kernel size is $4 * 4$ and padding is SAME. The third convolution layer output channel is 52, stride is 2 , convolution kernel size is $4 * 4$, and padding is SAME. The fourth convolution layer output channel is 104, stride is 1 , and convolution kernel size is $4 * 4$. The fifth convolution layer output channel is 1 , stride is 1 , convolution kernel size is 1 , and padding is SAME. The discriminator finally outputs a prediction map of $128 * 128 * 1$.

Figure 7 is a schematic diagram of the discriminator.

Figure 7

Schematic sketch of discriminator

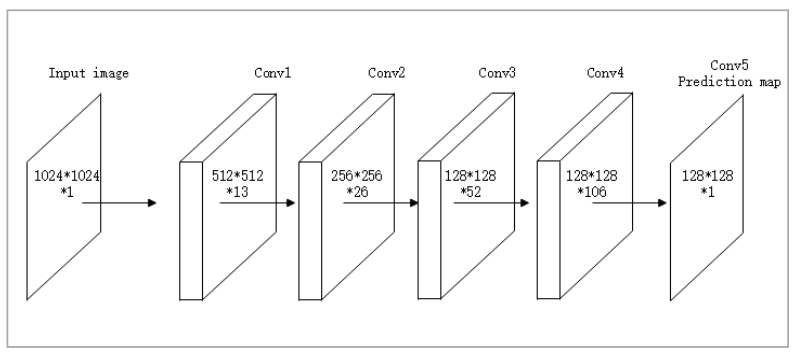

\subsection{Data Flow in Training Model}

The training of CycleGAN model is explained in Figure 8 as follows:
Figure 8

Dataflow of CycleGAN training

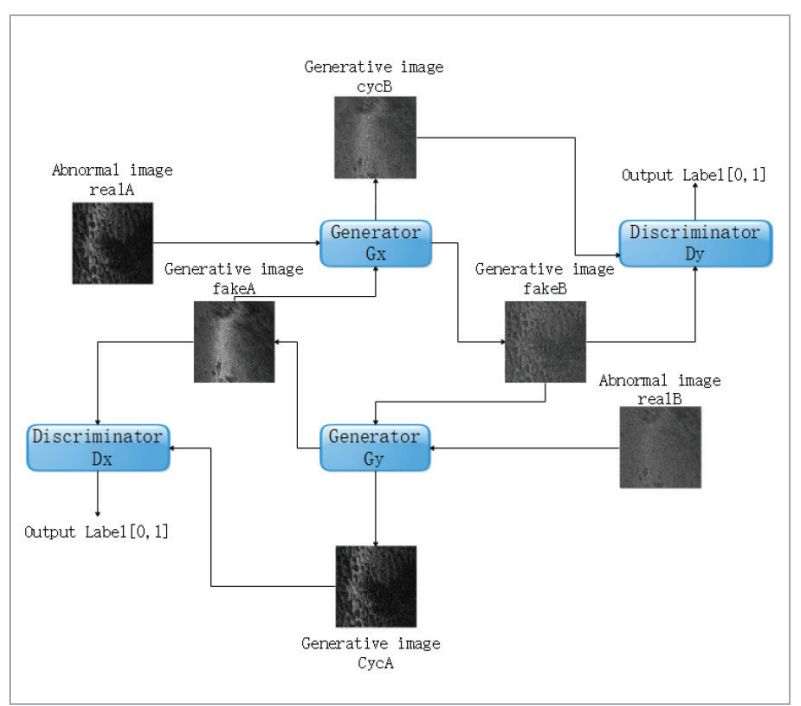

1 After inputting luminance abnormal image (class $X$ ) realA into generator $G_{X}$, and passing through generator network, the generated image fakeB is obtained.

2 FakeB is input into the discriminator $D_{X}$, and the category of fakeB is determined by the discriminator. If it belongs to the category $Y$ image (even brightness image), then outputs 1, otherwise 0.

3 FakeB is input into generator $G_{Y}$, and the generated image cycA is obtained after generator network.

4 The normal image ( $Y$ class, even brightness image) realB is input into the generator $G_{Y}$, and the generated image fakeA is obtained after generator network. 
5 FakeA is input into the discriminator $D_{Y}$, and the category of fakeA is determined by the discriminator. If it belongs to the category $Y$ image (even brightness image), then outputs 0 , otherwise 1 .

6 FakeA is input into generator $G_{X}$, and the generated image cycB is obtained after generator network.

7 According to the formulas derived in section 3 , the loss function value of the model is calculated to minimize the difference between fakeB and realB, fakeA and realA, cycA and realA, cycB and realB.

\section{Experimental Process and Results Analysis}

\subsection{Computer Configuration for Experiments}

CPU: i7-8700K; Memory: 64GB; GPU: NVIDIA RTX 2080Ti 11G; Deep learning framework: Tensorflow.

\subsection{Datasets Construction}

We cut 76 scenes images of Taklimakan Desert area into several subgraphs of $1024 * 1024$ size, screened 170 subgraphs with abnormal brightness as $X$ images and 260 normal images as $Y$ images. Figure 9 shows an example of a training set, where Figure 9(a) is a subgraph of an abnormal image with uneven brightness and Figure 9(b) is a subgraph of a normal image with even brightness.

\section{Figure 9}

Example of dataset: (a) abnormal brightness image;

(b) normal image

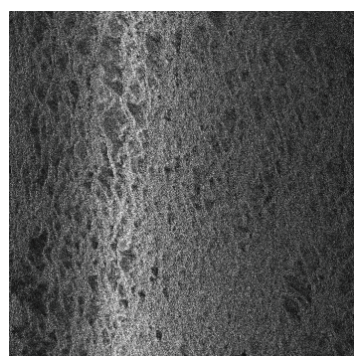

(a)

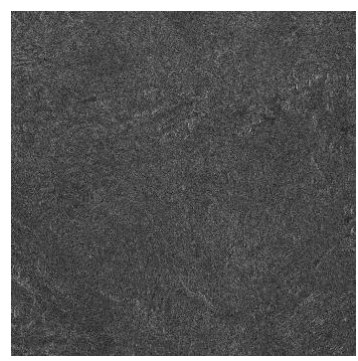

(b)

\subsection{CycleGAN Training}

When setting the learning parameters, we refer to the learning parameters adopted in references [42] and [18], and make reasonable modifications. The learning parameters are set as: batch size is 2 , the number of iterations is 200 , the initial learning rate is 0.0002 , the learning rate of the first 100 iterations is kept at 0.0002 , the learning rate of the last 100 iterations is linearly attenuated, and at the end of 200 training sessions, it dropped to 0 . The optimizer selects Adam algorithm. $\lambda$ in (6) is 5 .

\subsection{Experimental Results and Analysis}

After the model training, we select some abnormal brightness images to import into the model to obtain the output image, as shown in Figure 10, where Figure 10(a) is an abnormal brightness image and Figure 10 (b) is an image after brightness compensation of the CycleGAN model. Figure 11 is a horizontal profile line of Figure 10.

\section{Figure 10}

The result of proposed method: (a) original image;

(b) proposed method

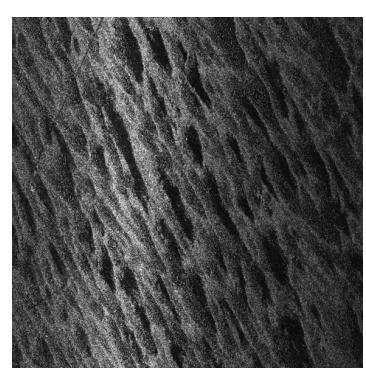

(a)

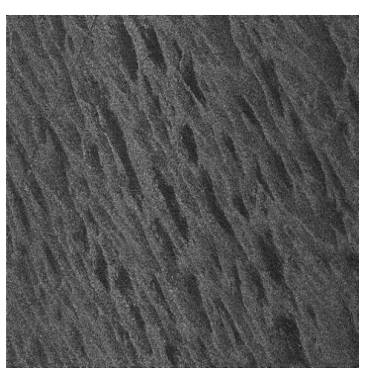

(b)
From Figure 10(a), it can be seen that the original image is bright on the left side and dark on the right side, with obvious "bright traces" and poor visual effect. After the processing of the proposed method, we get a better brightness even image. After analysis, we arrive at the following conclusions.

1 It can be seen from Figure 10 that the overall gray level of the image processed by the proposed method is basically the same and the brightness is moderate. There are obvious bright traces in the original image, and the processed image solves the problem of bright traces well.

2 It can be seen from Figure 11 that the horizontal profile line of the original image shakes violently, fluctuates obviously, and the gray values in the local area are too high and too low. These indicate 
Figure 11

Profile line of Figure 10

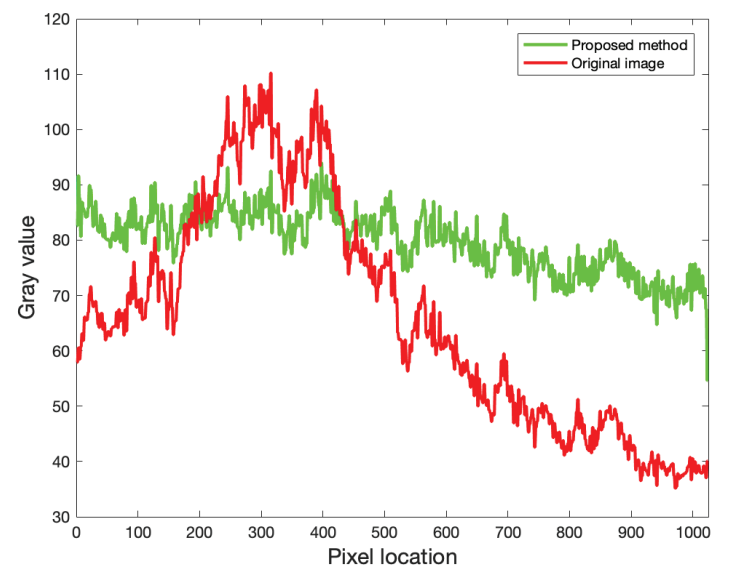

that the original image has a serious problem of uneven brightness. After the processing of our method, the horizontal profile line of the image is gentle, and the overall brightness is moderate. There is no phenomenon that the local brightness is too high or too low and the overall brightness is too dark or too bright, and the overall visual effect is good.

\section{Comparison Between the Proposed Method and the Classical Dodging Algorithms}

In order to fully verify the effectiveness of the proposed method, we use the histogram equalization,
MASK dodging algorithm, and Wallis dodging algorithm to compensate the brightness of the original images for comparison, and use brightness mean, standard deviation, and average gradient for comparative analysis.

\subsection{Comparison Between Histogram Equalization and the Proposed Method}

We use histogram equalization method and the proposed method to compensate the brightness of abnormal brightness image (Figure 12(a)), respectively. The processing results are shown in Figures 12(b)12 (c), respectively.

As can be seen from Figure 12, the brightness compensation effect of the proposed method is obviously better than that of the histogram equalization. From the perspective of visual effect, the image after histogram equalization (Figure 12(b)) still has obvious brightness imbalance problem, and the average brightness has been greatly improved compared with the original image, so the overall brightness of the processed image is not appropriate and the visual effect is poor. The proposed method can solve the problem of uneven brightness in the image, and the overall brightness is moderate and the overall visual effect is better.

\subsection{Comparison Between MASK Dodging Algorithm and the Proposed Method}

We use MASK dodging algorithm and the proposed method to compensate the brightness of abnormal brightness image (Figure 13(a)) respectively. The processing results are shown in Figures 13(b)-13(c).

Figure 12

Comparison of the results of histogram equalization and proposed method: (a) original image; (b) the result of histogram equalization; (c) the result of proposed method

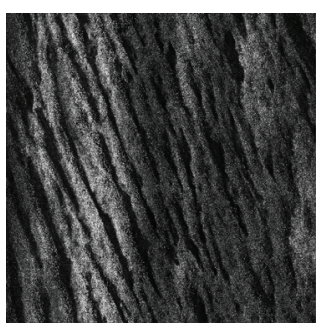

(a)

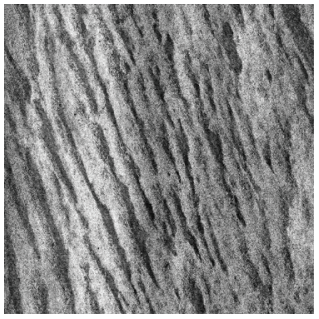

(b)

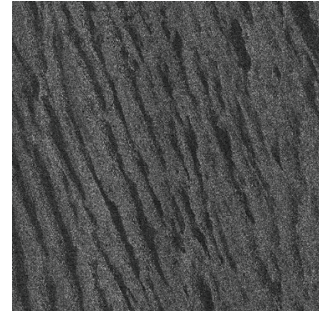

(c) 


\section{Figure 13}

Comparison of the results of MASK dodging and proposed method: (a) original image; (b) the result of MASK dodging; (c) the result of proposed method

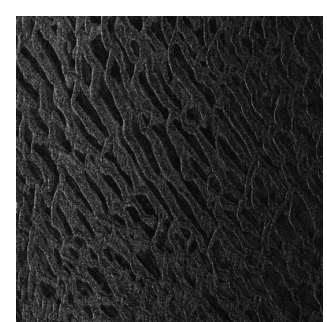

(a)

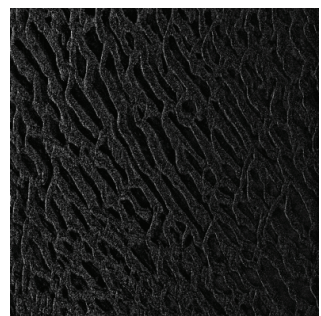

(b)

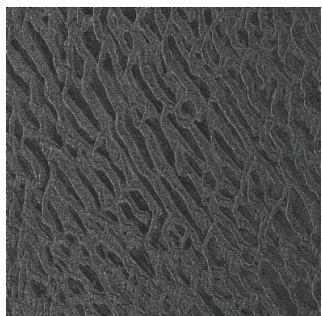

(c)

It can be seen from Figure 13 that the brightness compensation effect of the proposed method is better than that of the MASK dodging algorithm. The overall brightness of the image processed by MASK dodging algorithm is reduced, the visual effect is poor, and there is still local uneven brightness on the left side of the image. However, in the result of brightness compensation based on our method, the phenomenon of light trace has been well eliminated and overall brightness of the image is even, moreover there is no problem that the overall brightness is too dark or too bright, and the overall visual effect is good.

In order to further accurately evaluate the brightness compensation results, we cut the original image, MASK dodging results and the processing results of the proposed method into 9 image blocks, number them from left to right and from top to bottom (as shown in Figure 14), calculate the brightness mean value of each image block respectively, and draw the statistical results

\section{Figure 14}

Schematic sketch of image division and numbering

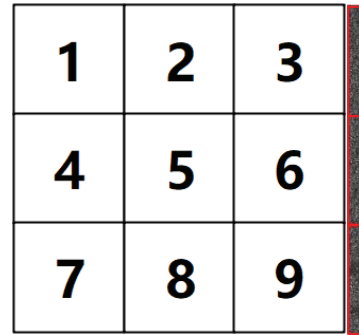

(a)

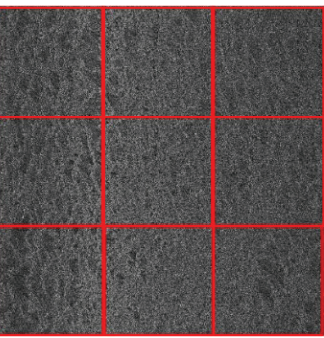

(b) into a line chart. The distribution curves of brightness mean value are shown in Figure 15.

\section{Figure 15}

Contrast chart of brightness mean

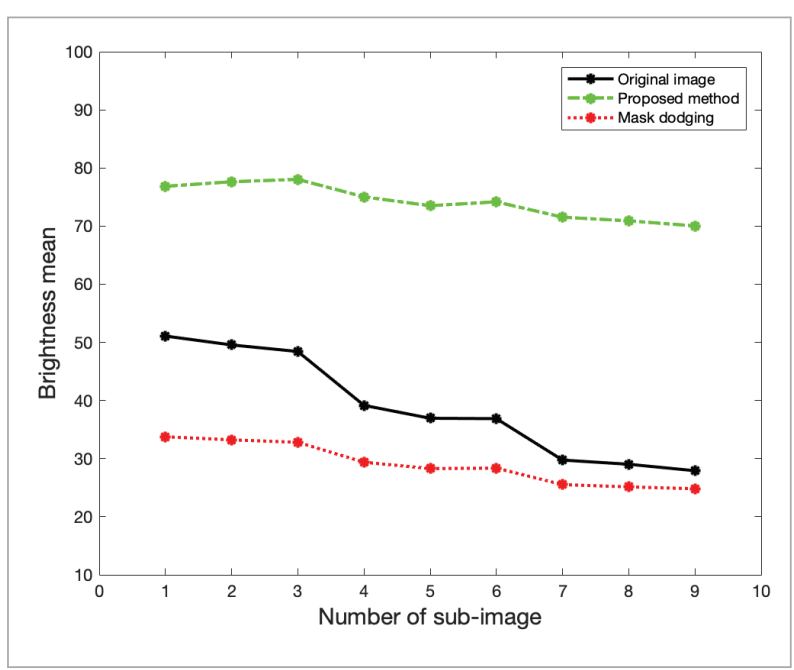

Through the analysis of the above chart, the following conclusion is drawn.

It can be seen from Figure 15 that the brightness mean curve of the original image fluctuates greatly, which indicates that there is serious uneven brightness phenomenon in the image. The brightness mean curve of the image processed by MASK dodging algorithm is smoother than that of the original image, which shows that the brightness of the image is more even to some extent. But there is still a phenomenon of uneven brightness and the overall gray values are small, 
which shows that the image is dark and the visual effect is poor. However, the brightness curve of the image processed by the proposed method is the smoothest, and the overall gray values are moderate. So our method not only solves the shortcomings of MASK dodging algorithm in the brightness compensation effect, but also solves the problems of dark brightness and poor visual effect after compensation.

\subsection{Comparison Between Wallis Dodging Algorithm and the Proposed Method}

We use Wallis dodging algorithm and the proposed method to compensate the brightness of the abnormal brightness image (Figure 16(a)). The processing results are shown in Figures 16(b)-Figure 16(c), respectively.

Table 1 shows two quantitative indexes of brightness means and standard deviations corresponding to Figure 16.

The brightness mean indicates the average level of image's gray value. If the brightness mean is moderate, the overall visual effect of the image is good. The brightness mean is defined as follows.

$$
\bar{x}=\frac{1}{n} \sum_{i=1}^{n} x_{i}
$$

where $n$ is the total number of samples, $x_{i}$ is the brightness value of the $i$ th sample.

Standard deviation represents the magnitude of fluctuations in data sets. The bigger the standard deviation is, the more dispersed the gray distribution of the image is, the larger the fluctuation range of the gray value is, and the greater the contrast of the image is. Standard deviation is defined as follows.

$$
\sigma=\sqrt{\frac{\sum_{i=1}^{n}\left(x_{i}-\bar{x}\right)^{2}}{n}},
$$

where $n$ is the total number of samples, $\bar{x}$ is the average gray level of the image, and $x_{i}$ is the $i$ th sample value. If the mean value of brightness is moderate, it means the overall visual effect of the image is moderate. Too high or too low mean value of brightness indicates poor visual effect of the image. The smaller the standard deviation is, the smaller the dynamic range of the gray value of the image is. In addition, the smaller the overall brightness difference of the image is, the more balanced and consistent brightness is. If the overall standard deviation of the image is too large, the brightness of the image is inconsistent, and there is a problem of uneven brightness.

\section{Table 1}

Brightness mean and standard deviation of original image and two algorithms

\begin{tabular}{l|c|c|c}
\hline $\begin{array}{c}\text { Quantitative } \\
\text { index }\end{array}$ & $\begin{array}{c}\text { Original } \\
\text { image }\end{array}$ & $\begin{array}{c}\text { Wallis } \\
\text { dodging }\end{array}$ & $\begin{array}{c}\text { Proposed } \\
\text { method }\end{array}$ \\
\hline $\begin{array}{l}\text { Brightness } \\
\text { mean }\end{array}$ & 75.19 & 126.05 & 80.39 \\
\hline $\begin{array}{l}\text { Standard } \\
\text { deviation }\end{array}$ & 68.29 & 68.36 & 65.38 \\
\hline
\end{tabular}

\section{Figure 16}

Comparison of the results of Wallis dodging and proposed method: (a) original image; (b) the result of Wallis dodging; (c) the result of proposed method

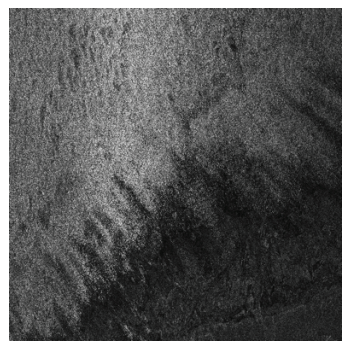

(a)

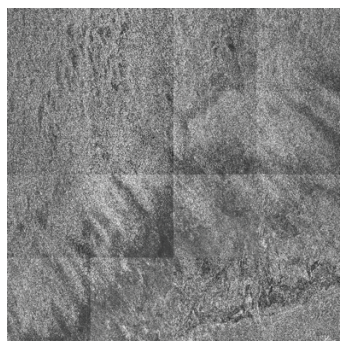

(b)

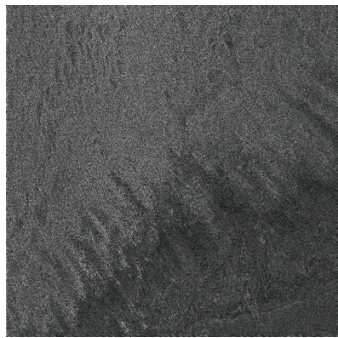

(c) 
Based on the analysis of the above figures and table, the following conclusions are drawn.

1 It can be seen from Figure 16 that Wallis dodging algorithm has achieved certain brightness compensation effect. However, the image is seriously distorted and there are pseudo stripes. Moreover, the overall image is too bright, the visual effect is poor, and a lot of information in the image is lost. This is because Wallis dodging algorithm itself has some problems such as overall hue deviation and "block effect", so the classic Wallis dodging algorithm cannot solve the problem of brightness compensation well.

2 It can be seen from Table 1 that the average brightness of the image processed by Wallis dodging algorithm is too high, which verifies the problem of "the overall image is too bright and the visual effect is not good" mentioned above. Secondly, the standard deviation of the image processed by Wallis is larger than that of one processed by the proposed method. It shows that the dynamic range of gray value of the image is large, the overall brightness of the image is inconsistent, and the brightness compensation effect is poor. The image processed by the proposed method has moderate average brightness, small standard deviation, balanced brightness, good compensation effect and better visual effect.

In addition, we also give the average gradient index to evaluate the effects of Wallis dodging algorithm and the method in this paper [37].

The average gradient reflects the details of the image and expresses the richness of the details of the image.
Generally, the bigger the average gradient of the image, the bigger the contrast of the details, the richer the image level, and the clearer the image. The average gradient is defined as follows.

$$
\nabla \bar{g}=\frac{1}{M N} \sum_{i=1}^{M} \sum_{j=1}^{N}\left[\Delta_{x} f(i, j)^{2}+\Delta_{y} f(i, j)^{2}\right]^{\frac{1}{2}},
$$

where $f(i, j)$ denotes the gray value of the image at $(i, j), \Delta_{x} f(i, j)$ and $\Delta_{y} f(i, j)$ denote the gradient of pixel $(i, j)$ in the direction of $x$ and $y, M$ denotes the number of rows of the image, and $N$ denotes the number of columns of the image.

To fully prove the universality of this method, we select another image for comparison, as shown in Figure 17. The average gradients of the three images in Figure 17 are calculated as shown in Table 2.

\section{Table 2}

Average gradient of original image and two algorithms

\begin{tabular}{|c|c|c|c|}
\hline $\begin{array}{c}\text { Quantitative } \\
\text { index }\end{array}$ & $\begin{array}{c}\text { Original } \\
\text { image }\end{array}$ & $\begin{array}{c}\text { Wallis } \\
\text { dodging }\end{array}$ & $\begin{array}{c}\text { Proposed } \\
\text { method }\end{array}$ \\
\hline Average gradient & 26.12 & 35.45 & 39.81 \\
\hline
\end{tabular}

As shown in Figure 17(b), the problems of "block effect" and hue shift are common in the image processed by Wallis dodging algorithm, and "block effect" can cause the image to appear blocks like pseudo stripes and introduce unnecessary details. The average gradient index reflects the amount of image details. For the sake of fairness, we cut sub-images of Figure 17

\section{Figure 17}

Comparison of the results of two algorithms: (a) original image; (b) the result of Wallis dodging; (c) the result of proposed method

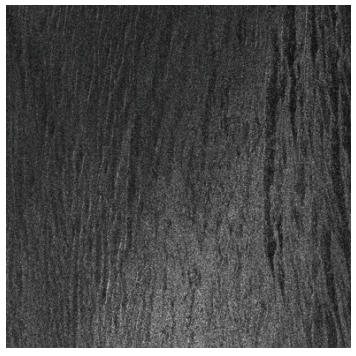

(a)

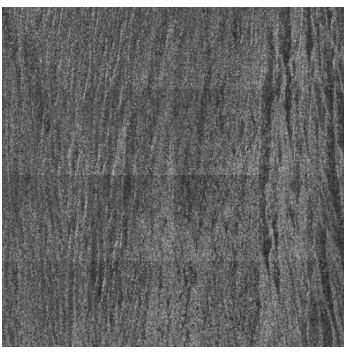

(b)

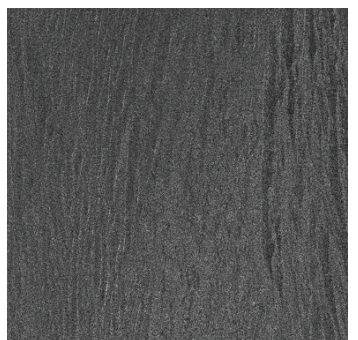

(c) 
into $4 * 4$ blocks along the pseudo stripes produced by "block effect" to avoid the interference of "block effect" on the evaluation index, and number them from left to right and from top to bottom, as shown in Figure 18. Then the average gradient of each image block is calculated, and the statistical results are drawn into a line chart, as shown in Figure 19.

\section{Figure 18}

Schematic sketch of image division and numbering

\begin{tabular}{|c|c|c|c|}
\hline 1 & 2 & 3 & 4 \\
\hline 5 & 6 & 7 & 8 \\
\hline 9 & 10 & 11 & 12 \\
\hline 13 & 14 & 15 & 16 \\
\hline
\end{tabular}

(a)

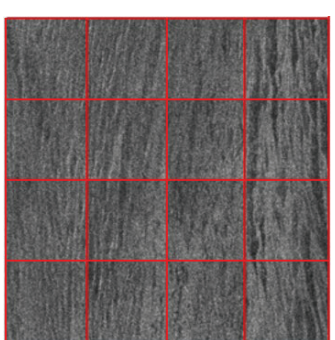

(b)
Figure 19

Contrast chart of average gradient

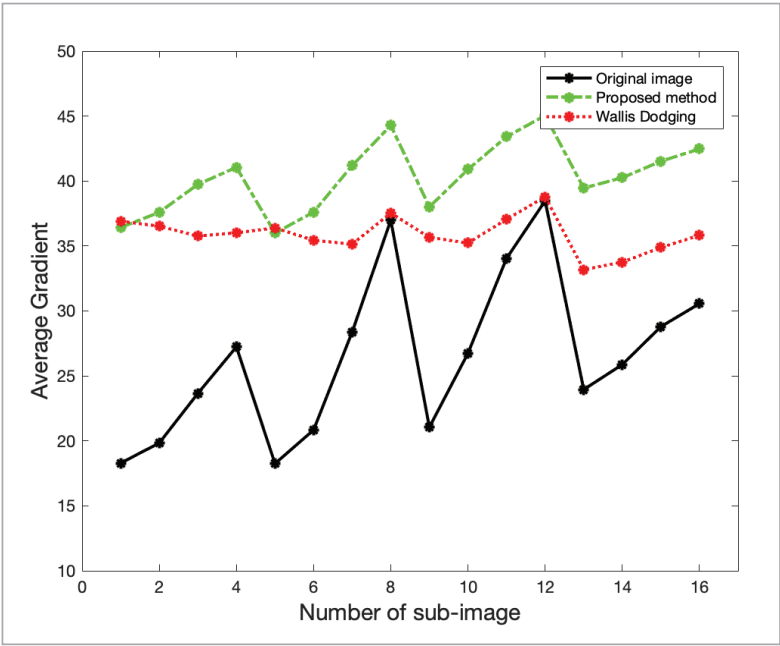

According to the analysis of Figure 17, Table 2 and Figure 19, we can draw the following conclusions.

1 Because of the "block effect" and hue shift in Wallis dodging algorithm, it is difficult to obtain an ideal brightness compensation image by Wallis dodging algorithm. The "block effect" introduces unnecessary information, and the hue shift causes the image to be lighter or darker. But the image processed by the proposed method can effectively solve the above problems.

2 It can be seen from Table 2 that the average gradient of the proposed method is greater than that of Wallis dodging algorithm, and both our method and Wallis dodging algorithm are greater than the average gradient of the original image. The results show that both of the two algorithms have a certain effect, but the image detail level processed by the proposed method is richer, and the brightness compensation effect is better.

3 It can be seen from Figure 19 that after the interference of "block effect" is eliminated, the average gradient of the 16 image blocks processed by the proposed method is still greater than the corresponding Wallis dodging algorithm processing result. This shows that our method is still better than Wallis method without "block effect", which proves that our method has better brightness compensation effect [28,1,35,2,6,7,14,15,-36].

\subsection{Comparison of Three Traditional Dodging Algorithms with the Proposed Method}

In order to better compare the performance of the above-mentioned traditional dodging algorithms and the proposed method, we put the MASK dodging algorithm, Wallis dodging algorithm, histogram equalization method, and the proposed method together for comparative experiments. The results are shown in Figure 20, and the corresponding quantitative indexes are compared in Table 3.

Table 3

Comparison of quantitative indexes of different methods

\begin{tabular}{l|c|c|c|c|c}
\hline $\begin{array}{c}\text { Quantitative } \\
\text { index }\end{array}$ & $\begin{array}{c}\text { Original } \\
\text { image }\end{array}$ & $\begin{array}{c}\text { MASK } \\
\text { dodging }\end{array}$ & $\begin{array}{c}\text { Wallis } \\
\text { dodging }\end{array}$ & $\begin{array}{c}\text { Histogram } \\
\text { equalization }\end{array}$ & $\begin{array}{c}\text { Proposed } \\
\text { method }\end{array}$ \\
\hline Brightness mean & 74.40 & 68.89 & 122.74 & 128.55 & 77.82 \\
\hline Standard deviation & 65.09 & 63.83 & 69.09 & 73.82 & 63.30 \\
\hline Average gradient & 32.45 & 36.20 & 39.98 & 40.11 & 40.44 \\
\hline
\end{tabular}




\section{Figure 20}

Comparison of the results of four algorithms: (a) original image; (b) the result of MASK dodging; (c) the result of Wallis dodging; (d) the result of histogram equalization; (e) the result of proposed method

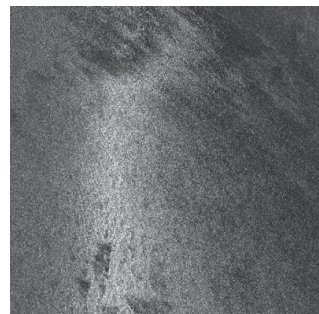

(a)

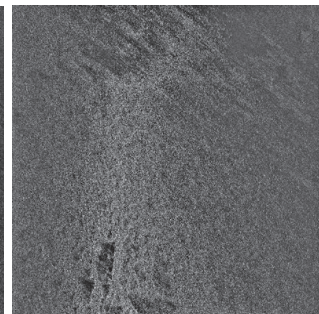

(b)

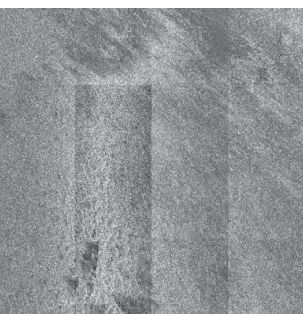

(c)

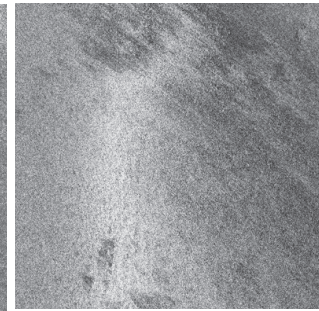

(d)

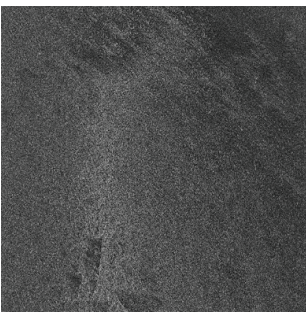

(e)
It can be seen from Figure 20 and Table 3 that the three traditional dodging algorithms and the method proposed in this paper have achieved certain dodging effect after image processing. However, the average brightness of Wallis and histogram equalization images is greater than 120 , while the original image is only 74.40 , which shows that the brightness of the images processed by the two methods is too large and the visual effect is poor. But the brightness mean values processed by the MASK dodging and the proposed method are moderate, and the visual effects are better. Among the two indexes of standard deviation and average gradient, the proposed method is the best among the four methods. Therefore, from the perspective of three indicators, the proposed method is obviously superior to the other three traditional methods.

In order to compare the computational efficiency of the proposed deep learning method and the traditional methods, we provide the processing time of 1,10 , and 100 SAR images. We perform the proposed method on the GPU of NVIDIA RTX 2080ti 11g and three traditional methods on the CPU of i5-8259u. The time taken is shown in Table 4.

\section{Table 4}

Comparison of computational efficiency of different methods

\begin{tabular}{l|c|c|c}
\multicolumn{1}{c|}{ Method } & 1 image & 10 image & 100 image \\
\hline Proposed Method & $0.30 \mathrm{~s}$ & $3.09 \mathrm{~s}$ & $31.53 \mathrm{~s}$ \\
\hline MASK dodging & $0.03 \mathrm{~s}$ & $0.36 \mathrm{~s}$ & $3.54 \mathrm{~s}$ \\
\hline Wallis dodging & $0.02 \mathrm{~s}$ & $0.18 \mathrm{~s}$ & $1.72 \mathrm{~s}$ \\
\hline Histogram equalization & $0.01 \mathrm{~s}$ & $0.17 \mathrm{~s}$ & $0.14 \mathrm{~s}$ \\
\hline
\end{tabular}

It can be seen that the time consumption of the three traditional methods for brightness compensation is less than that of the proposed method. This is because the computational complexity of deep learning is far greater than that of the traditional methods, and an ordinary deep learning model can also have hundreds of millions of parameters. In the brightness compensation problem discussed in this paper, we pay more attention to the processing effect of algorithm rather than the time consumption [21, $24,25,26,29,30,31,36]$.

\section{Conclusions}

In this paper, a brightness compensation method is proposed for GF-3 SAR images with uneven brightness based on CycleGAN. Firstly, the image is cut into several subgraphs of $1024 * 1024$. Then the images with uneven brightness are classified as $X$ images and the images with even brightness are classified as $Y$ images. Then an improved CycleGAN model suitable for SAR images is constructed. Finally, the final model is obtained by training with the learning parameters provided in this paper. Results of brightness compensation show that, the proposed method is better than the traditional dodging algorithms. The overall visual effect of the images processed by the proposed method has been greatly improved, which solves the problem of uneven brightness of GF-3 SAR images, and has strong practical value in GF-3 SAR data processing. 


\section{Acknowledgments}

This work was supported by the National Natural Science Foundation of China (No. 61102163), the Fundamental Research Funds for the Central Universities (No. GK201903085) and the State Key Lab- oratory of Geo-Information Engineering (No. SKLGIE2019-M-3-5). The authors would like to thank China Centre for Resources Satellite Data and Application for providing some GF-3 SAR images to do brightness compensation experiments in this paper.

\section{References}

1. Afza, F., Khan, M. A., Sharif, M., Kadry, S., Manogaran, G., Saba, T., Damaševičius, R. A Framework of Human Action Recognition Using Length Control Features Fusion and Weighted Entropy-Variances Based Feature Selection. Image and Vision Computing, 106. https:// doi.org/10.1016/j.imavis.2020.104090

2. Bai, Z., Li, Y., Chen, X., Yi, T., Wei, W., Wozniak, M., Damaševičius, R. Real-Time Video Stitching for Mine Surveillance Using a Hybrid Image Registration Method. Electronics (Switzerland), 2020, 9(9), 1-18. https:// doi.org/10.3390/electronics9091336

3. Cheng, X. Y., Xie, L., Zhu, J. X., Hu, B., Shi, Q. Review of Generative Adversarial Network. Computer Science, 2019, 46(3), 74-81.

4. Chen, T. Q., Schmidt, M. Fast Patch-Based Style Transfer of Arbitrary Style. arXiv preprint arXiv: 1612.04337, 2016.

5. Chen, H., Guo, W., Yan, J. W. Synthetic Aperture Radar Image Target Segmentation Method Based on Boundary and Texture Information. Journal of Image and Graphics, 2019, 24(6), 882-889.

6. Damaševičius, R., Maskeliūnas, R., Narvydas, G., Narbutaitè, R., Połap, D., Woźniak, M. Intelligent Automation of Dental Material Analysis Using Robotic Arm with Jerk Optimized Trajectory. Journal of Ambient Intelligence and Humanized Computing, 2020, 11(12), 62236234. https://doi.org/10.1007/s12652-020-02605-8

7. Dong, G., Wei, W., Xia, X., Woźniak, M., Damaševičius, R. Safety Risk Assessment of a pb-zn Mine Based on Fuzzy-Grey Correlation Analysis. Electronics (Switzerland), 2020, 9(1). https://doi.org/10.3390/electronics9010130

8. Fei, P. Dodging Research for Remote Sensing Image. M.S. thesis, University of Chinese Academy of Science, Beijing, China, 2013.

9. Fan, C., Chen, X. S., Zhong, L., Zhou, M., Shi, Y., Duan, Y. L. Improved Wallis Dodging Algorithm for Large-Scale Super-Resolution Reconstruction Remote Sensing Im- ages. Sensors, 2017, 17(3), 623. https://doi.org/10.3390/ s17030623

10. Goodfellow, I. J., Pouget-Abadie, J., Mirza, M., Xu, B., Warde-Farley, D., Ozair, S., Courville, A., Bengio, Y. Generative Adversarial Nets. In Proceedings of Advances in Neural Information Processing Systems, Montreal, Canada, 2014, 2672-2680.

11. Hu, X., Yao, Q. L., Hou, B. Q., Song, H. J., Lei, H. Target Recognition Using Convolution Neural Network for SAR Images. Science Technology and Engineering, 2019, 19(21), 228-232.

12. Isola, P., Zhu, J. Y., Zhou, T., Efros, A. A. Image-to-Image Translation with Conditional Adversarial Networks. In Proceedings of IEEE Conference on Computer Vision and Pattern Recognition, Venice, Italy, 2017, 1125-1134. https://doi.org/10.1109/CVPR.2017.632

13. Johnson, J., Alahi, A., Li, F. F. Perceptual Losses for Real-Time Style Transfer and Super-Resolution. In Proceedings of European Conference on Computer Vision, Amsterdam, Netherlands, 2016, 694-r11. https://doi. org/10.1007/978-3-319-46475-6_43

14. Jouhari, H., Lei, D., Al-qaness, M. A. A., Abd Elaziz, M., Damaševičius, R., Korytkowski, M., Ewees, A. A. Modified Harris Hawks Optimizer for Solving Machine Scheduling Problems. Symmetry, 2020, 12(9). https:// doi.org/10.3390/sym12091460

15. Khan, M. A., Ashraf, I., Alhaisoni, M., Damaševičius, R., Scherer, R., Rehman, A., Bukhari, S. A. C. Multimodal Brain Tumor Classification Using Deep Learning and Robust Feature Selection: A Machine Learning Application for Radiologists. Diagnostics, 2020, 10(8). https://doi.org/10.3390/diagnostics10080565

16. Li, T., Lu, J. National Orthophoto Map of radar Remote Sensing with GF-3. Beijing, China, 2018, 20, http://www. sasmac.cn/xwfb/zxdt/201809/t20180920_408025.html.

17. Lin, Y. L., Dai, X. Y., Li, L., Wang, X., Wang, F. Y. The New Frontier of AI Research: Generative Adversarial Networks. Acta Automatica Sinica, 2018, 44(5), 7775-792. 
18. Liu, Z. L., Zhu, W., Yuan, Z. Y. Image Instance Style Transfer Combined with Fully Convolutional Network and cyclegan. Journal of Image and Graphics, 2019, 24(8), 1283-1291.

19. Lauraitis, A., Maskeliūnas, R., Damaševičius, R., Krilavičius, T. Detection of Speech Impairments Using Cepstrum, Auditory Spectrogram and Wavelet Time Scattering Domain Features. IEEE Access, 2020, 8, 96162-96172. https://doi.org/10.1109/ACCESS.2020.2995737

20. Niu, B., Wu, P., Ma, L., Liu, J. W. A Behavior Data Set Extension Method Based on Generative Adversarial Network. Computer Technology and Development, 2019, 29(7), 43-48.

21. Nisa, M., Shah, J. H., Kanwal, S., Raza, M., Khan, M. A., Damaševičius, R., Blažauskas, T. Hybrid Malware Classification Method Using Segmentation-Based Fractal Texture Analysis and Deep Convolution Neural Network Features. Applied Sciences (Switzerland), 2020, 10(14). https://doi.org/10.3390/app10144966

22. Oliver, C., Quegan, S. Understanding Synthetic Aperture Radar Images. Boston, MA, USA: Artech House, 1998.

23. Orsini, G., Ramponi, G., Carrai, P., Di Federico, R. A Modified Retinex for Image Contrast Enhancement and dynamic control. In Proceedings of IEEE International Conference on Image Processing, Barcelona, Spain, 2003, 393-396.

24. Olanrewaju, L., Oyebiyi, O., Misra, S., Maskeliunas, R., Damasevicius, R. Secure ear Biometrics Using Circular Kernel Principal Component Analysis, Chebyshev Transform Hashing and Bose-Chaudhuri-Hocquenghem Error-Correcting Codes. Signal, Image and Video Processing, 2020, 14(5), 847-855. https://doi.org/10.1007/ s11760-019-01609-y

25. Orujov, F., Maskeliūnas, R., Damaševičius, R., Wei, W. Fuzzy Based Image Edge Detection Algorithm for Blood Vessel Detection in Retinal Images. Applied Soft Computing Journal, 2020, 94. https://doi.org/10.1016/j. asoc.2020.106452

26. Ryselis, K., Petkus, T., Blažauskas, T., Maskeliūnas, R., Damaševičius, R. Multiple Kinect Based System to Monitor and Analyze Key Performance Indicators of Physical Training. Human-Centric Computing and Information Sciences, 2020, 10(1). https://doi. org/10.1186/s13673-020-00256-4

27. Son, J., Park, S. J., Jung, K. H. Retinal Vessel Segmentation in Fundoscopic Images with Generative Adversarial Networks. arXiv preprint arXiv: 1706.09318, $201 \%$.
28. Sun, Z., Zhao, G., Woźniak, M., Scherer, R., Damaševičius, R. Bankline Detection of GF-3 SAR Images Based on Shearlet. Peer Journal of Computing, 2021, Sci. 7:e611 Doi: $10.7 r 17 /$ peerj-cs.611.

29. Sahlol, A. T., Elaziz, M. A., Jamal, A. T., Damaševičius, R., Hassan, O. F. A Novel Method for Detection of Tuberculosis in Chest Radiographs Using Artificial Ecosystem-Based Optimisation of Deep Neural Network Features. Symmetry, 2020, 12(7). https://doi.org/10.3390/ sym12071146

30. Sahlol, A. T., Yousri, D., Ewees, A. A., Al-qaness, M. A. A., Damaševičius, R., Elaziz, M. A. COVID-19 Image Classification Using Deep Features and Fractional-Order Marine Predators Algorithm. Scientific Reports, 2020, 10(1). https://doi.org/10.1038/s41598-020-71294-2

31. Sun, Z., Lin, D., Wei, W., Wozniak, M., Damaševičius, R. Road Detection Based on Shearlet for GF-3 Synthetic Aperture Radar Images. IEEE Access, 2020, 8, 2813328141. https://doi.org/10.1109/ACCESS.2020.2966580

32. Wang, T., Yu, H. F., Liu, J., Zhang, Q. J. System Technologies of Spaceborne Dual-Antenna InSAR. Spacecraft Engineering, 2016, 25(6), 6-12.

33. Wang, M., Pan, J. A method of removing the uneven illumination for digital aerial image. Journal of Image and Graphics, 2004, 9(6), 744-748.

34. Wang, B. S. Method of Automatic Registration and Mosaic for SAR Images. Ph.D. dissertation, Wuhan University, Wuhan, China, 2015.

35. Wei, W., Sun, Z. G., Zhang, Z. H., Scherer, R., Damaševičius, Robertas. Improved Fisher MAP Filter for Despeckling of High-Resolution SAR Images Based on Structural Information Detection. Journal of Internet Technology, 2021, 22(2): 413-421. DOI: 10.3966/160'792642021032202016.

36. Wieczorek, M., Silka, J., Polap, D., Wozniak, M., Damaševičius, R. Real-Time Neural Network Based Predictor for Cov19 Virus Spread. PLoS ONE, 2020, 15(12). https://doi.org/10.1371/journal.pone.0243189

37. Xu, Y. Research on Algorithms for Super-Resolution Image Reconstruction. Ph.D. dissertation, Beijing University of Posts and Telecommunications, Beijing, China, 2013.

38. Yi, Z. L., Zhang, H., Tan, P., Gong, M. L. DualGAN: Unsupervised Dual Learning for Image-to-Image Translation. In Proceedings of IEEE International Conference on Computer Vision, Venice, Italy, 2017, 28-49. https:// doi.org/10.1109/ICCV.2017.310 
39. Zhang, Q. J. System Design and Key Technologies of the GF-3 Satellite. Acta Geodaetica et Cartographica Sinica, 2017, 46(3), 269-27\%.

40. Zhang, Q. J., Liu, J., Li, Y., Qi, Y. L., Zhao, L. B. System Design Verification of GF-3 Satellite. Spacecraft Engineering, 2017, 26(5), 1-7.

41. Zhu, X. C., Tang, G. J. A Survey on Generative Adversarial Networks in Image Processing. Journal of Nanjing University of Posts and Telecommunications (Natural Science Edition), 2019, 39(3), 1-12.

42. Zhu, J. Y., Park, T., Isola, P., Efros, A. A. Unpaired Image-to-Image Translation using Cycle-Consistent Adversarial Networks. In Proceedings of IEEE In- ternational Conference on Computer Vision, Venice, Italy, 2017, 2242-2251. https://doi.org/10.1109/ ICCV.2017.244

43. Zhu, X. X., Dong, Z., Yu, A. X., Wu, M. Q., Li, D. X., Zhang, Y. S. New Approaches for Robust and Efficient Detection of Persistent Scatterers in SAR Tomography. Remote Sensing, 2019, 11(3), 356. https://doi.org/10.3390/ rs11030356

44. Zhu, X. X., He, F., Ye, F., Dong, Z., Wu, M. Q. Sidelobe Suppression with Resolution Maintenance for SAR Images via Sparse Representation. Sensors, 2018, 18(5), 1589. https://doi.org/10.3390/s18051589 